\title{
A SEXUALIDADE DA MULHER ACOMETIDA COM O CÂNCER DE MAMA
}

\author{
SEXUALITY OF WOMEN AFFECTED WITH BREAST CANCER
}

\author{
Cintia Barcala de Oliveira Santos ${ }^{1}$, Ivana Maria Passini Sodré Siviero ${ }^{2,3}$, Gisele Acerra \\ Biondo Pietrafesa ${ }^{3}$ \\ ${ }^{1}$ Instituto de Responsabilidade Social Sírio Libanês - Lucy Montoro, Mogi-Mirim, SP, \\ Brasil; ${ }^{2}$ Faculdade Municipal Professor Franco Montoro, Mogi-Guaçu, SP, Brasil; ${ }^{3}$ Centro \\ Universitário Unipinhal, Espírito Santo do Pinhal, SP, Brasil.
}

\section{RESUMO}

Objetivo: A sexualidade é um bem-estar físico, mental, emocional, cultural, religioso e social. O câncer de mama pode provocar na mulher diversas mudanças, comprometendo sua sexualidade. O objetivo deste trabalho é compreender como o câncer de mama afeta a sexualidade feminina. Métodos: Para tal foi utilizado o Female Sexual Function Index (FSFI), um instrumento que visa avaliar a resposta sexual feminina nas últimas quatro semanas, contendo seis domínios. Participaram da pesquisa 20 mulheres acometidas com o câncer de mama, num ambulatório de Oncologia de um Hospital Municipal do interior do Estado de São Paulo. Resultados: A escala prevê um escore máximo de 36 pontos e disfunçáo sexual em mulheres que obtém abaixo ou igual a 26 pontos. Nessa pesquisa $100 \%(\mathrm{n}=20)$ das mulheres apresentaram valor abaixo do escore considerado normal, o que revelou a presença da disfunção sexual. Conclusão: todas as 20 (100\%) mulheres entrevistadas apresentaram, sentimentos de vergonha, desânimo, frustração, alteraçóes na sexualidade, necessitando assim de uma assistência humanizada, envolvendo uma somatória de aspectos físicos, psicológicos, sociais, culturais, espirituais e econômicos.

Descritores: Qualidade de vida; câncer de mama; sexualidade.

\section{ABSTRACT}

Objective: The sexuality is a physical, mental, emotional, cultural, religious and social well-being. The breast cancer can cause many changes in women, compromising her sexuality. The goal of this job is to understand how breast cancer affects female sexuality. Methods: For that, it was used the "Female Sexual Function Index (FSFI), an instrument whose objective is to rate the female sexual answer of the last four weeks, containing six domains. Results: Twenty women with breast cancer has participated from the research, in an oncologist ambulatory from a municipal hospital in the countryside of São Paulo. The scale predicts a maximum score of 36 points and sexual disfunction in women who gets it lower or equal 26 points. In the research $100 \%$ of women have demonstrated sexual disfunction. Conclusion: The 20 (100\%) of the women interviewed presented feelings of shame, discouragement, frustration, changes in sexuality, thus requiring humanized assistance, involving a sum of physical aspects, psychological, social, cultural, spiritual and economic.

Descriptors: Quality of life; breast cancer; sexuality. 


\section{INTRODUÇÃO}

De acordo com Camargo e Neto ${ }^{1}$ a sexualidade foi definida pela Organização Mundial da Saúde (OMS) como:

Uma energia que nos motiva para encontrar amor, contato, ternura e intimidade; ela integra-se no modo como sentimos, movemos, tocamos e somos tocados, é ser-se sensual e ao mesmo tempo ser-se sexual. A sexualidade influencia pensamentos, sentimentos, açôes e interaçóes e, por isso, influência também a nossa saúde física e mental.

A sexualidade é melhor definida como um bem-estar físico, mental, emocional, cultural, religioso e social. É abrangente de múltiplos aspectos e constantes modificaçóes durante todo o ciclo da vida ${ }^{2}$.

A sexualidade abrange todos os aspectos físicos e mentais com o intuito de alcançar a satisfação sexual, seja consigo mesma ou com o outro, despertando sua identidade e assim contribuindo para a reprodução da humanidade ${ }^{3}$.

A Associação Psiquiátrica Americana definiu um esquema para o ciclo de resposta sexual, sendo este composto de quatro fases. Para que o casal tenha satisfaçáo, tanto o homem como a mulher necessitam completar essas fases. Sáo elas o desejo (fantasias acerca da atividade sexual e desejo de exercê-la); a excitaçáo (sensação subjetiva de prazer sexual e mudanças fisiológicas decorrentes); o orgasmo (ápice de prazer sexual); a resolução (sensaçấo geral de bem-estar e relaxamento) $)^{4}$.

Quando alguns dos aspectos associados à sexualidade são comprometidos, a mulher apresenta dificuldades em responder ou experimentar o prazer sexual, levando a um quadro de disfunção sexual ${ }^{5}$. Nas disfunçôes sexuais femininas a falta de interesse pelo sexo é um dos maiores desafios ${ }^{6}$.

O câncer de mama é a consequência de uma multiplicação desordenada das células e que se reproduzem rapidamente, originando o aparecimento de tumores ou neoplasias malignas, podendo provocar metástases ${ }^{7}$.

O câncer de mama pode provocar na mulher diversas mudanças em seu pensamento, desde o seu diagnóstico, sinais e sintomas, adaptaçáo com a doença, recuperação, reabilitação e no seu processo de morte. Provoca alteraçôes psicossociais náo somente no doente, como em todos que estão envolvidos ${ }^{8}$.

Desde o primeiro contato com a doença, as mulheres e seus familiares, passam por angústia, esta pode vir acompanhada por vários sintomas, dentre eles, depressão, estresse constante, frustraçáo, perda da autoestima, privacidade, liberdade e identidade, alteraçôes na qualidade de vida?

A palavra câncer soa muito forte, ainda mais quando falamos no câncer de mama, sendo esse muito temido, porque afeta uma regiáo considerada a identidade feminina ${ }^{10}$. A 
mama é a figura da feminilidade, uma vez sendo comprometida a mulher pode começar a se perguntar sobre seus valores, sua autoestima em relação a seu corpo e sexualidade ${ }^{11}$.

Os tratamentos do câncer de mama são agressivos, causando sequelas que afetam a sexualidade e particularmente a vida sexual. Os procedimentos podem ser cirúrgicos, assim alterando a aparência, sensibilidade e funcionalidade das mamas. As terapêuticas, quimioterapia, radioterapia e terapia hormonal ocasionam efeitos colaterais variados como náusea, vomito, fadiga, alopecia, menopausa induzida, redução da lubrificação vaginal, redução da excitação sexual, dispaurenia e anorgasmia ${ }^{12}$.

A qualidade de vida do doente deve ser uma responsabilidade contínua dos profissionais de saúde, preocupando-se como esse indivíduo reage à sua saúde e a outros aspectos de sua vida diária, podendo assim conhecer seus valores, desejos e proporcionar um bem-estar em suas atividades simples como à vida diária. Sua cura nem sempre é possível, mas alguns aspectos da doença podem ser controlados se o profissional reconhecer e utilizar as melhores estratégias no cuidar. A humanização do profissional de enfermagem contribui para uma reabilitação adequada, ter uma boa comunicação, diálogo, criar laços de confiança, podendo esclarecer as dúvidas sexuais, reduzir medos e preocupaçôes dos doentes oncológicos ${ }^{13}$.

Dessa forma, esse trabalho tem como objetivo compreender se o câncer de mama afeta a sexualidade feminina.

\section{MATERIAIS E MÉTODOS}

Foram entrevistadas 20 pacientes do sexo feminino do setor de Oncologia de um Hospital Municipal localizado no interior do Estado de São Paulo. O critério de inclusão foi mulheres que estivessem em tratamento para o câncer de mama e o de exclusão, pacientes masculinos, as pacientes que estivessem em tratamento de outros tipos de câncer e aquelas que não tiveram interesse em participar do estudo.

Para a coleta de dados foi estipulado um prazo de 1 semana, sendo do dia 02 ao dia 06 do mês de setembro/2019, visando obter pacientes diagnosticadas com câncer de mama, que estavam, em tratamento na oncologia.

Foi aplicado um formulário de identificação e o Female Sexual Function Index (FSFI) ${ }^{14}$, um instrumento com dezenove questóes, que visa avaliar a resposta sexual feminina nas últimas quatro semanas, contendo seis domínios: desejo sexual, excitação sexual, lubrificação vaginal, orgasmo, satisfação sexual e dor para respondê-lo ${ }^{14}$.

Cada questão apresenta um padrão de resposta que varia de 0 a 5 , sendo em forma crescente em relação à presença da função questionada, exceto nas questóes sobre o domínio da dor que a pontuação é invertida. Ao final da aplicação um escore total é apresentado, sendo a soma de escores de cada domínio multiplicada por um fator que 
homogeneíza a influência de cada domínio no escore total. $\mathrm{O}$ ponto de corte do escore total é definido como 26 para a população de origem do instrumento, sendo assim será possível identificar disfunção sexual a partir de valores iguais ou abaixo desse ponto ${ }^{14}$. O Domínio Desejo nas questóes 1 e 2, tem a variação de escore de 1 a 5, fator de multiplicação é 0,6, escore mínimo de 1,2 e escore máximo 6,0. Domínio Excitação nas questóes 3, 4, 5 e 6 tem a variação de escore de 0 a 5, fator de multiplicação é 0,3, escore mínimo de 0,0 e escore máximo 6,0. Domínio Lubrificação nas questôes 7, 8, 9 e 10 tem a variação de escore de 0 a 5, fator de multiplicação é 0,3 , escore mínimo de 0,0 e escore máximo 6,0. Domínio Orgasmo nas questôes 11, 12 e 13 tem a variação de escore de 0 a 5, fator de multiplicação é 0,4 , escore mínimo de 0,0 e escore máximo 6,0. Domínio Satisfação na questão 14 tem a variação de escore de 0 a 5, nas questóes 15 e 16 tem a variação de escore de 1 a 5, fator de multiplicaçáo é 0,4, escore mínimo de 0,8 e escore máximo 6,0. Domínio Dor nas questões 17,18 e 19 tem a variação de escore de 0 a 5, fator de multiplicação é 0,4 , escore mínimo de 0,0 e escore máximo 6,0. Escore total mínimo 2,0 e escore total máximo 36,0.

O trabalho foi submetido à Plataforma Brasil e avaliado pelo CEP da Faculdade Municipal Professor Franco Montoro. A coleta de dados ocorreu após aprovação do estudo pelo comitê (CAAE 17438719.9.0000.5425). O estudo foi explicado às participantes e o termo de consentimento livre e esclarecido foi lido e orientado, sendo solicitada a assinatura das participantes da pesquisa.

\section{RESULTADOS E DISCUSSÃO}

O presente trabalho contou com a participação de 20 pacientes em acompanhamento na oncologia para o tratamento do câncer de mama, que responderam um formulário de entrevista, a fim de avaliar a resposta sexual feminina nas últimas quatro semanas.

Quanto às características sociodemográficas das participantes, observa-se na Tabela 1, o predomínio na faixa etária maior que 42 anos (70\%) e estado civil casada (75\%) e nível de escolaridade baixo (analfabeto a ensino fundamental completo somaram 50\%).

Na tabela 2, ao analisar o número de parceiros, observa-se que $80 \%$ das mulheres têm parceiro único e quanto à frequência sexual, durante o mês, $60 \%$ relataram que não apresenta relaçóes sexuais.

De acordo com Silva ${ }^{15}$, pacientes com bloqueio do desejo sexual, apresentam ansiedade e sentimento de fuga do parceiro. Podendo explicar a inatividade sexual das participantes. 
Tabela 1. Distribuição do número de mulheres segundo características sociodemográficas. Mogi Guaçu, São Paulo, Brasil, 2019 ( $\mathrm{n}=20)$.

\begin{tabular}{lcc}
\multicolumn{1}{c}{ VARIÁVEIS } & N & $\%$ \\
\hline Faixa etária & 3 & 15 \\
$18-36$ & 3 & 15 \\
$37-42$ & 14 & 70 \\
$>42$ anos & & \\
\hline Estado civil & 3 & 15 \\
$\quad$ Solteira & 15 & 75 \\
Casada & 1 & 5 \\
$\quad$ Divorciada & 1 & 5 \\
$\quad$ Viúva & & \\
Escolaridade & 2 & 10 \\
$\quad$ Analfabeto & 7 & 35 \\
$\quad$ Ensino fundamental incompleto & 7 & 5 \\
$\quad$ Ensino fundamental completo & 1 & 40 \\
$\quad$ Ensino médio completo & 8 & 10 \\
$\quad$ Superior & 2 &
\end{tabular}

Fonte: Dados da pesquisa atual

Tabela 2. Distribuição do número de mulheres segundo número de parceiros e frequência sexual. Mogi Guaçu, São Paulo, Brasil, 2019 ( $\mathrm{n}=20)$.

\begin{tabular}{ccc} 
VARIÁVEIS & $\mathbf{N}$ & $\%$ \\
\hline Número de parceiros & & \\
0 & 3 & 15 \\
1 & 16 & 80 \\
2 & 1 & 5 \\
\hline Frequência Sexual & & \\
Ausente & 12 & 60 \\
1 X semana & 2 & 10 \\
A cada 15 dias & 4 & 20 \\
1 X mês & 2 & 10 \\
\hline
\end{tabular}

Fonte: Dados da pesquisa atual

Na Tabela 3, ao analisar os resultados do FSFI, nota-se que 20 mulheres (100 \%) apresentaram valor abaixo do escore considerado normal, o que revelou a presença da disfunção sexual.

Tabela 3. Disfunção sexual feminina. Mogi Guaçu, São Paulo, Brasil, 2019 ( $\mathrm{n}=20)$.

\begin{tabular}{ccc} 
DISFUNCÃO SEXUAL & $\mathbf{N}$ & $\%$ \\
\hline$\leq 26$ & 20 & 100 \\
$>26$ & 0 & 0 \\
\hline
\end{tabular}

Fonte: Dados da pesquisa atual 
Ao analisar os domínios separadamente (Tabela 4), observa-se que há prejuízo em todos eles, já que a pontuação máxima possível é 6,0 e a média de nota é menor que 3,0 em todos os domínios, mostrando que as mulheres não estão satisfeitas com sua função sexual, tendo dificuldades em atingir o orgasmo e estando possivelmente mais propensas a sentirem dor. A função sexual está associada à saúde emocional feminina, o interesse e a satisfação sexual estão ligados à expressividade emocional ${ }^{16}$.

O corpo da mulher passa por mudanças devido à queda do estrogênio, favorecendo alteraçôes nas fases da resposta sexual ${ }^{17}$. $\mathrm{Na}$ amostra estudada teve um predomínio de mulheres com mais de 42 anos, fase em que algumas podem estar entrando no climatério.

A resposta sexual feminina sofre influências de aspectos psicológicos, uso de medicamentos, álcool ou drogas, sociocultural, sendo assim, qualquer alteração pode surgir disfunçōes sexuais ${ }^{15}$.

Os transtornos sexuais dolorosos crônicos são frequentes, o estresse e as condiçóes psicológicas têm desencadeado as condiçôes dolorosas. Por inibição da paciente e a falta de investigação não é feito o diagnóstico. Conhecer esse transtorno auxilia na detecção e tratamento precocemente ${ }^{18}$.

Entre as voluntárias entrevistadas, uma minoria fala com naturalidade sobre suas dificuldades sexuais, ainda existe receio. Das 20 participantes, 08 estiveram sexualmente ativas nesse mês. Um ponto importante apresentado, foi que 07 mulheres optaram, em comum acordo com seus parceiros, ficarem inativas sexualmente durante todo o tratamento, devido desconfortos, como dispareunia e ausência de lubrificação, sendo esses responsáveis pela inatividade, por esse motivo essa inatividade sexual, mesmo sendo uma opção, não pode ser julgada como uma escolha da mulher e sim como uma saída em frente a tantos medos, podendo observar nessas mulheres, baixos escores de satisfação com a vida sexual, variando de 0,8 a 2,0. Rodrigues et al ${ }^{19}$ relatam que mesmo quando o casal tem uma vida sexual satisfatória antes da doença, os efeitos após diagnóstico e tratamento pode desorganizar a vida conjugal. Uma mulher por crença religiosa optou pela abstinência sexual, por não aceitar métodos contraceptivos, outra também por crença religiosa não está ativa sexualmente, pois aguarda o casamento. Uma viúva, refere não ter atividade sexual. Uma mulher divorciada relata que devido sua crença náo pode mais ter parceiro. Uma participante solteira por opção, refere não ter vida sexual ativa. 
Tabela 4. Comparação dos escores mínimos e máximos e das médias dos domínios do FSFI. Mogi Guaçu, São Paulo, Brasil, 2019 ( $\mathrm{n}=20)$.

\begin{tabular}{lccc} 
DOMíNIOS & MÍN & MÁX & MÉDIA \\
\hline Desejo & 1,2 & 4,2 & 2,43 \\
Excitação & 0 & 4,5 & 1,245 \\
Lubrificação & 0 & 4,5 & 0,975 \\
Orgasmo & 0 & 5,6 & 1,58 \\
Satisfação & 0,8 & 5,2 & 2,84 \\
Dor & 0 & 5,6 & 1,44 \\
\hline
\end{tabular}

Fonte: Dados da pesquisa atual

A sexualidade está relacionada a tabus e preconceitos, sem o conhecimento, o profissional torna-se despreparado. A falta de informaçóes e conteúdo acadêmico, não estudado durante suas formaçóes iniciais, impede que o profissional da saúde colabore com o cuidado e a melhora da qualidade de vida dos pacientes ${ }^{20}$.

De acordo com Nepumuceno, Melo e Silva ${ }^{21}$, a sexualidade é um processo complexo e próprio de cada indivíduo, indo além do biológico, sendo o ato sexual fisiológico e a sexualidade como uma totalidade do ser humano. Estes autores ao abordarem o paciente com lesão na medula espinhal, relatam que não necessariamente há perda da sexualidade, visto que não se perde a sexualidade por causa de uma doença ou lesão.

Segundo Coimbra e Teixeira ${ }^{22}$, quando perguntado a pacientes portadores de diabetes, qual o profissional de saúde a quem recorrem primeiro para tratar sobre a sexualidade, o Enfermeiro náo é o profissional recorrido para tratar desse assunto e quanto a abordagem do Enfermeiro, referem que são acolhidos de forma superficial.

É fundamental uma atuação mais eficiente, seguindo um roteiro de exame físico, utilizando instrumentos como o NANDA-I (Diagnósticos de Enfermagem), onde o Domínio 8 Sexualidade está presente, contendo diagnóstico de disfunção sexual e padrão de sexualidade ineficaz ${ }^{23}$.

A abordagem da sexualidade deve acontecer antes do tratamento, para que as pacientes sejam informadas sobre os efeitos colaterais sexuais comuns relacionados ao tratamento e durante e pós a terapêutica para que sejam levantadas as dificuldades, que podem ser angustiantes e prejudicar a qualidade de vida. Entretanto, na visão dos enfermeiros as barreiras para essa intervenção se dão por falta de conhecimento e de habilidade para discutir e abordar o tema, mas também por vergonha, desconforto, questôes culturais e religiosas. ${ }^{24}$

Resende e Sobral ${ }^{25}$ em estudo sobre o ensino da sexualidade nos cursos de graduação em enfermagem observaram que os docentes se sentem despreparados para lidar com a temática por não terem tido também uma formação nessa área do conhecimento durante a graduação. Observa-se, dessa forma, um círculo vicioso em que o resultado é o 
pouco preparo técnico e a dificuldade de superar tabus impostos por questóes religiosas e culturais na formação pessoal e profissional dos enfermeiros.

\section{CONSIDERAÇÓES FINAIS}

O estudo apresentou como limitação um número (n) pequeno de sujeitos, embora tenham sido entrevistadas todas as mulheres que estavam em tratamento no setor de oncologia, dessa forma o resultado náo pode ser generalizado e deve ser replicado com outras mulheres nas mesmas condições. Mogi Guaçu, São Paulo, Brasil, 2019 ( $n=20)$. As mulheres acometidas com o câncer de mama desenvolvem desconforto sexual durante e ao término do tratamento, o que interfere na sua qualidade de vida, altera seu sentimento, emoçóes e traz insegurança quanto sua imagem.

Considerando os efeitos físicos e os aspectos psicossociais, há um aumento da disfunção sexual quando comparado às mulheres acometidas com o câncer de mama.

Todas as $20(100 \%)$ das mulheres entrevistadas apresentaram, sentimentos de vergonha, desânimo, frustração, alterações na sexualidade. Essas mulheres necessitam de uma assistência humanizada, envolvendo uma somatória de aspectos físicos, psicológicos, sociais, culturais, espirituais e econômicos. Este consiste em favorecer um ambiente confortável e um vínculo de afeto entre quem cuida e quem é cuidado, indo em busca de valores e desejos que se perdeu desde o diagnostico até a terapêutica. Essas mulheres precisam expressar seus sentimentos, vivências, necessidades, dúvidas, assim o profissional pode se direcionar as orientaçôes e intervençôes de acordo com a especificidades de cada mulher.

Uma avaliação precoce sobre a saúde sexual pode melhorar a qualidade de vida e a possível reabilitação sexual. Conhecer as necessidades dessas mulheres, para prestar uma assistência com qualidade, favorecer intervençóes que venham minimizar os efeitos do desconforto sexual, sendo assim há uma necessidade em mudar a prática assistencial.

Uma ação educativa é fundamental para capacitar os pacientes em tratamento a viverem com suas limitaçóes, assim visando a harmonia entre doença e tratamento. Estudos demonstraram que estratégias formais de abordagem do tema, com comunicação efetiva com casais sobre a prática sexual, através de aconselhamento telefônico, workshops, grupos operativos, resultaram em avaliaçóes positivas pelos participantes. Intervençôes com casais foram eficazes na promoção da comunicação, ajustes sexuais e relações funcionais por propiciarem melhor compreensão do diagnóstico e seus efeitos relacionados à sexualidade. ${ }^{24}$ Dessa forma, é recomendado que os serviços estabeleçam programas formais de intervenção, com preparo dos profissionais de saúde, pois Varssão et al ${ }^{24}$ observaram resposta prospectiva duradoura no comportamento dos enfermeiros que 
receberam aprimoramento profissional nesse quesito, demonstrando que os enfermeiros sentiram-se mais confiantes em iniciar as discussóes com os pacientes.

\section{REFERÊNCIAS}

Camargo SAP, Neto LFS. Sexualidade e gênero. Rev. Fac. Ciênc. Méd. de Sorocaba. 2017; 19(4):165-6.

Verenhitach BD, Medeiros JN, Elias S, Nazário ACP. Câncer de mama e seus efeitos sobre a sexualidade: uma revisão sistemática sobre abordagem e tratamento. Femina. 2014; 42(1).

Vieira EM, Santos DB, Santos MA, Giani A. Vivência da sexualidade após o câncer de mama: estudo qualitativo com mulheres em reabilitação. Rev. Latino-Am. Enfermagem. 2014; 22(3):408-14.

Fleury HJ, Pantaroto HSC, Abdo CHN. Sexualidade em oncologia. Diagn. Tratamento. 2011; 16(2):86-90.

Barreto APP, Nogueira A, Teixeira B, Brasil C, Lemos A, Lôrdelo P. O impacto da disfunção sexual na qualidade de vida feminina: um estudo observacional. Rev. Pesq. Fisio. 2018; 8(4):511-517.

Fleury HJ, Abdo CHN. Excitação sexual feminina subjetiva. Diagn Tratamento. 2018; 23(2):66-9.

Ribeiro VC, Portella SDC, Malheiro ES. Mulheres de meia idade e o enfrentamento do câncer de mama. Rev. Cuid. 2014; 5(2):799-805.

Otani MAP, Barros NF, Marin MJS. A experiência do câncer de mama: percepções e sentimentos de mulheres. Revista Baiana de Enfermagem. Salvador. 2015; 29(3):229239.

Gontijo IBR, Ferreira CB. Sentimentos de mulheres jovens ao diagnóstico de câncer de mama feminino. Revista Ciência \& Saúde. Porto Alegre. 2014; 7(1):2-10.

Almeida TG, Comassetto I, Alves KMC, Santos AAP, Silva JMO, Trezza MCSF. Vivência da mulher jovem com câncer de mama e mastectomizada. Escola Anna Nery Revista de Enfermagem. Rio de Janeiro. 2015; 19(3):432-438.

Prates ACL, Junior RF, Prates MFO, Veloso MF, Barros NM. Influência da imagem corporal em mulheres submetidas a tratamento para câncer de mama. Rev. Bras. Ginecol. Obstet. 2017; 39:175-183.

Santos DB, Santos MA, Vieira EM. Sexualidade e câncer de mama: uma revisão sistemática da literatura. Saúde Soc. São Paulo. 2014; 23(4):1342-1355. 
Soares SGSC, Albuquerque JOL. Intervenção do enfermeiro no tratamento quimioterápico de mulheres com câncer de mama. Revista Saúde em Foco. Teresina. 2014; 1(1) art. 3:29-45.

Pacagnella RC, Martinez EZ, Vieira EM. Validade de construto de uma versão do Female Sexual Function Index. Cad. Saúde Pública. Rio de Janeiro. 2009; 25(11):2333-2344.

Silva VA. Curso: Sexualidade humana. Universidade Candido Mendes AVM Faculdade Integrada. Rio de Janeiro. 2015

Antônio JZ, Silva A, Costa PPB, Jung D, Pereira CF, Nunes EFC, Latorre GFS. Funçáo sexual feminina, desgaste emocional por insatisfaçáo sexual e inteligência emocional. Rev. Fisioterapia Brasil. 2016; 17(6):544-550.

Correia LS, Brasil C, Silva MD, Silva DFC, Amorim HO, Lordêlo P. Função sexual e qualidade de vida de mulheres: um estudo observacional. Rev. Port. Med. Geral. Fam. 2016; 32:405-9.

Brasil APA, Abdo CHN. Transtornos sexuais dolorosos femininos. Diagn. Tratamento. 2016; 21(2):89-92.

Rodrigues NS, Orsini MRCA, Tertuliano IW, Bartholomeu D, Machado AA, Montiel JM. O impacto da mastectomia na sexualidade da mulher. Lecturas: Educación Física y Deportes. 2018; 23(242).

Rezende AV, Sobral OJ. As temáticas relativas à sexualidade humana na formação superior do profissional de enfermagem. Revista Científica FacMais. 2016; 5(1):25-39.

Nepumuceno E, Melo AS, Silva SS. Alterações relacionadas aos aspectos da sexualidade no lesado medular: revisão integrativa. Rev. Enferm. UFPE on line. Recife. 2014; $8(2): 396-406$.

Coimbra L, Teixeira ER. Percepção de homens com diabetes mellitus sobre sexualidade. Cienc. Cuid. Saude. 2015; 14(1):970-977.

Diagnósticos de enfermagem da NANDA-I: definições e classificação 2018-2019. NANDA Internacional. 11. ed. Porto Alegre. Artmed; 2018.

Vassão FV, Barbosa LR, Moraes GM, De Domenico EBL. Abordagem da sexualidade no cuidado ao paciente oncológico: barreiras e estratégias. Acta Paul. Enferm. 2018; 31(5):564-71.

Rezende AV, Sobral OJ. As temáticas relativas à sexualidade humana na formação superior do profissional de enfermagem. Revista Científica FacMais. 2016; 5(1): 25 39. 
Autor Correspondente: Cintia Barcala de Oliveira Santos

E-mail: barcalinhasantos@hotmail.com

Recebido em: 2020-06-14

Aprovado: 2020-11-11 